\title{
Efecto Antiangiogénico del Ácido Cafeico y Pinocembrina en el Proceso de Angiogénesis Fisiológica de Fetos de Pollo
}

\author{
Anti-Angiogenic Effect of Caffeic Acid and Pinocembrin in the \\ Process of Physiological Angiogenesis of Chicken Fetuses
}

Patricia Olivares O. ${ }^{\text {; }}$ María Paz Riquelme ${ }^{1}$; Pedro Leiva A. ${ }^{1,2}$; Luis A. Salazar ${ }^{3}$; Dina Guzmán-Oyarzo ${ }^{3}$ \& Carlos Rosas C. $^{2}$

Olivares, O. P.; RIQUelMe, M. P.; LEIVA, A. P.; SAlAZAR, L. A.; GUZMÁN-OYARZO, D. \& ROSAS, C. C. Efecto antiangiogénico del ácido cafeico y pinocembrina en el proceso de angiogénesis fisiológica de fetos de pollo. Int. J. Morphol., 38(1):135139, 2020.

RESUMEN: La angiogénesis es el proceso por el cual se forman nuevos vasos sanguíneos a partir de otros ya existentes. Para que esto se lleve a cabo de forma correcta debe existir un balance entre los factores proangiogénicos y los factores antiangiogénicos dentro del microambiente tisular. Por otra parte, la existencia de productos químicos naturales como los polifenoles, que son capaces de adquirirse en la dieta, inducen a estos factores a intervenir en el proceso de angiogénesis. Se administraron los polifenoles en filtros de metilcelulosa sobre la membrana alantocoriónica de huevos White Leghorn, manteniendo el posterior desarrollo normal del feto. Se utilizaron 15 fetos de pollo fijados en formalina tamponada, a los cuales se extrajo el corazón. El procesamiento de las muestras de corazón se realizó a través de técnicas histológicas, histoquímicas e inmunohistoquímica. Finalmente se evaluó la presencia del VEGF y la capacidad de formar vasos sanguíneos bajo el tratamiento con los polifenoles. La inmunorreactividad fue cuantificada mediante Image J®. Los resultados indican que Ácido cafeico y Pinocembrina disminuyen la densidad microvascular y la expresión de VEGF en corazones de fetos de pollo tratados con estos polifenoles. Tanto el Ácido Cafeico como la Pinocembrina cumplen un rol inhibitorio en el proceso de angiogénesis fisiológica en corazón de pollo, pudiendo modular las vías de señalización mediadas por los VEGFR o modulando la disponibilidad de VEGF. Estos polifenoles podrían utilizarse para el estudio de otros tejidos asociados a angiogénesis patológica.

PAlAbraS CLAVE: Angiogénesis; Polifenoles; Ácido Cafeico; Pinocembrina.

\section{INTRODUCCIÓN}

La angiogénesis es el proceso por el cual se forman nuevos vasos sanguíneos a partir de otros ya existentes, esto gracias a la acción de las células endoteliales que recubren la superficie interna de los vasos para ayudar al crecimiento y la reparación del tejido, que migran y proliferan, organizándose hasta formar estructuras tubulares que eventualmente se unirán, para finalmente madurar en vasos sanguíneos estables (Fakhrejahani \& Toi, 2012). La angiogénesis fisiológica ocurre durante el desarrollo embrionario y fetal y, en el adulto, durante el crecimiento esquelético, el ciclo menstrual y el embarazo (Chung \& Ferrara, 2011). También se reactivará en respuesta a estímulos fisiológicos durante la cicatrización de heridas y la inflamación (Li et al., 2003), y después de un injerto de tejido o isquemia (Chung et al., 2010).
Un balance entre los mediadores químicos que la promueven (factores proangiogénicos) y los que la inhiben (factores antiangiogénicos) dentro del microambiente tisular es necesario para el correcto funcionamiento de la angiogénesis. El principal estímulo proangiogénico es el factor de crecimiento endotelial vascular (VEGF); el cual pertenece a una familia compuesta por cinco proteínas homodiméricas. La proteína más importante es VEGF-A que regula la angiogénesis y la permeabilidad vascular mediante la activación de dos receptores el VEGFR-1 y VEGFR-2. La vía de señalización de VEGF se ha establecido como el principal regulador de la angiogénesis, en donde las células endoteliales expresan el receptor 2 del factor de crecimiento endotelial vascular (VEGFR-2), un receptor de tirosina quinasa que impulsa positivamente las

\footnotetext{
${ }^{1}$ Escuela de Tecnología Médica, Facultad de Ciencias de la Salud, Universidad San Sebastián, Santiago, Chile

${ }^{2}$ Departamento de Ciencias Morfológicas, Facultad de Medicina y Ciencia, Universidad San Sebastián, Santiago, Chile.

${ }^{3}$ Centro de Farmacogenética y Biología Molecular, Núcleo de Biorecurso Tecnológico y Científico (BIOREN), Universidad de La Frontera, Temuco, Chile.
} 
respuestas mitogénicas y quimiotácticas de las células endoteliales a VEGF. La migración de las células depende de un gradiente de VEGF, mientras que la proliferación está regulada por la concentración de VEGF (Gerhardt et al., 2003).

A su vez, existen los polifenoles, que son una clase estructural de productos químicos orgánicos principalmente naturales, que se caracterizan por la presencia de uno o más anillos aromáticos que llevan uno o más grupos hidroxilo (Manach et al., 2004; Ververidis et al., 2007). Dependiendo del número de anillos de fenol y los elementos estructurales que unen estos anillos entre sí, generalmente se clasifican como ácidos fenólicos, flavonoides, estilbenos, lignanos, secoiridoides, entre otros (Diniz et al., 2017). Estos los podemos adquirir a través de la dieta, por lo cual el consumo de frutas y verduras se correlaciona con la disminución del riesgo de desarrollar enfermedades de alta prevalencia, como las enfermedades cardiovasculares y el cáncer (Birt et al., 2001; Mitjavila \& Moreno, 2012). La evidencia indica que los polifenoles son capaces de alterar las cascadas de señalización involucradas en los pasos iniciales de la angiogénesis, así como durante los eventos que conducen a la formación de estructuras capilares (Diniz et al.).

El ácido cafeico (AC) es un tipo de ácido fenólico, se encuentra en una variedad de alimentos como: tomates, zanahorias, arándanos, vino, té, café, entre otros (Wang et al., 2016). Ahn et al. (2009), informaron que el ácido cafeico tiene actividad antiangiogénica y antioxidante en fibroblastos humanos, células endoteliales y tumorales; se examinó el efecto inhibitorio en la formación de tubos de HUVECs en el cual el ácido cafeico tuvo un fuerte efecto inhibitorio sobre la formación de tubos, perturbó la morfología del tubo causando una ligera fragmentación de la red, además inhibió casi por completo el alargamiento de las células e indujo la muerte celular de la mayoría de ellas. Por otro lado, pinocembrina (PC) es uno de los flavonoides más importantes aislados de miel y propóleos. En este mismo estudio, la PC tuvo un efecto inhibitorio leve en la formación de tubos, y muy pocos efectos inhibidores en la proliferación celular (Ahn et al.). También se ha reportado que PC posee actividad antiinflamatoria, antimicrobiana y antioxidante (Saad et al., 2015), las cuales podrían tener gran interés farmacológico debido a que pueden ser utilizadas en la terapia de varias enfermedades cáncer y/o enfermedades vasculares (Rasul et al., 2013).

Si bien a la fecha existen estudios, que demuestran las propiedades antiangiogénicas de AC y PC, no se han llevado a cabo estudios en donde se analice el efecto del tratamiento con estos polifenoles sobre la formación de vasos en órganos tan importantes como el corazón de fetos de pollo.

\section{MATERIAL Y MÉTODO}

Material biológico y grupos experimentales. Se utilizaron 15 fetos de pollo los cuales fueron tratados con dos polifenoles: ácido cafeico (PM: $180,16 \mathrm{~g} / \mathrm{mol}$ ) y pinocembrina (PM: $256,25 \mathrm{~g} / \mathrm{mol}$ ) a diferentes concentraciones. El tratamiento se realizó a través de la abertura de una ventana en la cáscara del huevo para posteriormente depositar los filtros de metilcelulosa embebidos con los polifenoles sobre la membrana alantocorionica de pollo (MAC).

Los fetos fueron distribuidos en 3 grupos de la siguiente manera:

Control: 4 fetos en condiciones normales con DMSO.

Grupo 1: 7 fetos que reciben $10 \mu \mathrm{L}$ de ácido cafeico [50 $\mu \mathrm{M}]$.

Grupo 2: 4 fetos que reciben $10 \mu \mathrm{L}$ de pinocembrina [10 $\mu \mathrm{M}]$.

Una vez eutanasiados, fueron fijados en formalina tamponada durante dos semanas. Posteriormente, se realizó una toracotomía para extraer el corazón de los fetos de pollo (entre 14-18 días de desarrollo embriofetal) los cuales fueron separados para cada grupo experimental, para luego dejarlos nuevamente en la formalina tamponada durante 16 horas. Finalmente se realizó el procesamiento histológico a través de la obtención de cortes de $4 \mu \mathrm{m}$ de espesor y posterior tinción con Hematoxilina/Eosina y Tricrómico de Arteta.

\section{Cuantificación de la intensidad de inmunotinción de}

VEGF. Para la cuantificación de VEGF los cortes de corazón de cada grupo experimental fueron desparafinados e hidratados previamente para luego ser recuperados antigénicamente por incubación por 30 minutos en solución Dako Target Retrieval ${ }^{\circledR}$. Luego fueron tratados en Peróxido de Hidrógeno al $3 \%$ en metanol por 10 minutos. Para la detección las muestras fueron incubadas con un anticuerpo policlonal de conejo Anti-VEGF (Clone ABS82) (Millipore, USA), en una dilución 1:400, durante 30 minutos a $37^{\circ} \mathrm{C}$. Para el revelado se utilizó el kit "Histostain-Plus Broad Spectrum Kit", HRP (Invitrogen) y 3,3'-Diaminobenzidina (DAB). Como control positivo se utilizaron cortes de placenta. Se observaron 20 campos de cada grupo, y se tomaron microfotografías con ayuda de un Microscopio Óptico (Motic BA310) en aumento 400x. Se utilizó el programa Image J para cuantificar la intensidad de inmunotinción, de acuerdo a lo descrito por Rosas et al. (2014) 
Comparación de la densidad microvascular. Se realizó la tinción Tricrómica de Arteta (Rosas et al., 2013) en los tejidos de corazón, se contaron los vasos sanguíneos en las muestras histológicas de cada grupo, utilizando un Microscopio Óptico (Motic BA310) con un aumento de 400x. El conteo se realizó en 25 campos microscópicos en forma ciega por dos examinadores. Los resultados se expresaron como $\mathrm{N}^{\circ}$ de vasos sanguíneos/por campo. Finalmente se tomaron microfotografías de cada campo.

Análisis estadístico. Se utilizó el análisis de varianza (ANOVA) para comparación entre grupos experimentales, con prueba de Tukey para comparaciones múltiples. Se consideró diferencia significativa si el valor de $p<0,05$. Los resultados se expresaron como promedio \pm error estándar. Para el cálculo estadístico se utilizó el Software Graph Pad Prism 5.0.

\section{RESULTADOS}

Se analizó la expresión de VEGF mediante su inmunomarcaje en células endoteliales del tejido histológico del corazón de pollo con tinción citoplasmática (Fig. 1A).

Se observó una disminución de la expresión de VEGF en los grupos tratados con AC y PC. Al comparar AC y PC no hubo diferencia significativa $(p=0,1049)$.

Cuando se comparó la densidad microvascular en los distintos grupos experimentales (control v/s tratados), se observó una disminución significativa del número de vasos sanguíneos tanto para el grupo tratado con AC y PC. Para el grupo tratado con AC se observó 7,44 $\pm 5,32$ vasos sanguí-

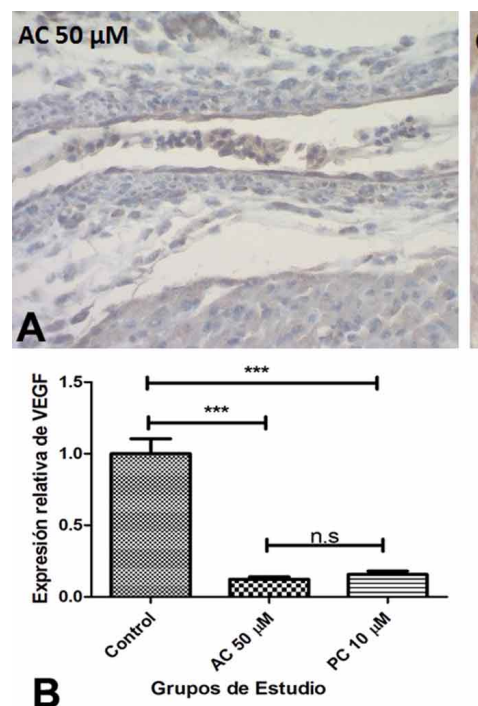

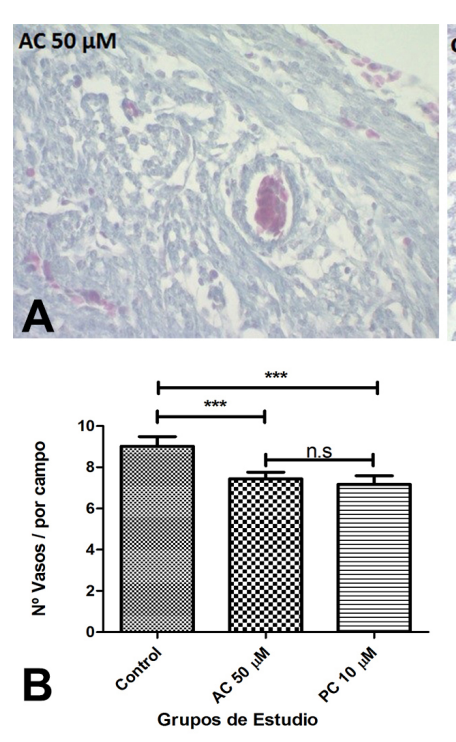

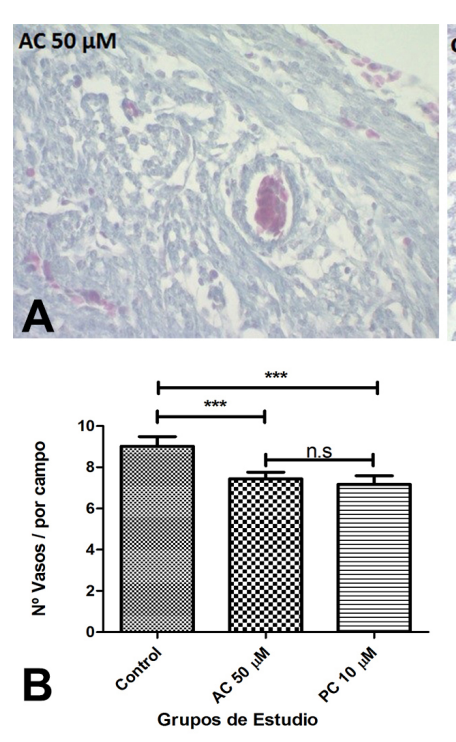

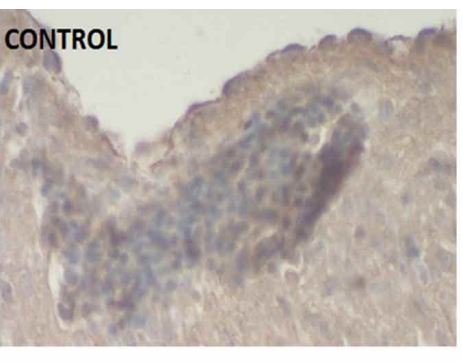

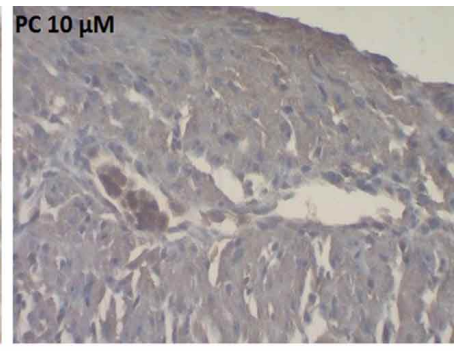

Fig. 1. Expresión de VEGF en corazón de pollo. A) Imagen representativa de corazón de pollo que muestran la presencia de VEGF mediante inmunohistoquímica. Marcación de VEGF con patrón de tinción citoplasmático. (Control, AC, PC). B) Cuantificación del nivel de expresión de VEGF. Los datos se comparan con respecto al grupo control. (ns: no hay diferencia entre $\mathrm{AC}$ y $\mathrm{PC} ; \mathrm{p}=0,1049$ )
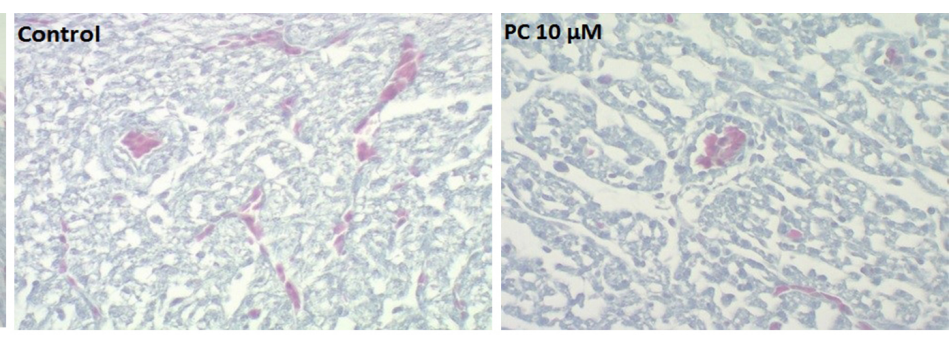

Fig. 2. Cuantificación de densidad microvascular en corazón. A) Imagen representativa de corazón con Tricrómico de Arteta, para facilitar el conteo de vasos sanguíneos. (Control, AC, PC). B) Número de vasos por campo en cada grupo; Control, AC [50 $\mu \mathrm{M}]$ y PC $[10 \mu \mathrm{M}]$ : Entre los grupos AC y $\mathrm{PC}$ no hubo alguna significancia. Hubo diferencia estadísticamente significativa ( $\mathrm{p}<0.0001)$ al comparar: Control vs AC $[50 \mu \mathrm{M}]$ y Control vs PC $[10 \mu \mathrm{M}]$, Al comparar AC $[50 \mu \mathrm{M}]$ vs PC [10 $\mu \mathrm{M}]$, no hubo significancia estadística, ns: $\mathrm{p}=0,3047$. 
neos/campo y para el grupo tratado con PC se observó 7,17 \pm 0,41 vasos sanguíneos/campo (Fig. 2). En tanto que para el grupo control el conteo fue de 9,02 \pm 0,46 vasos sanguíneos/campo. (Fig. 1B)

Por otra parte, al comparar el grupo de AC con PC no se observan diferencias significativas $(\mathrm{p}=0,3047)$.

\section{DISCUSIÓN}

Según el grado de inmunotinción de VEGF, se establece que existen diferencias estadísticamente significativas entre los grupos control/AC, control/PC, y no existen diferencias significativas entre el grupo AC/PC. Esto podría ser explicado porque ambos polifenoles presentan distinta biodisponibilidad y mecanismo de acción en las vías de señalización asociadas a angiogénesis. Moyle et al. (2015), demostraron que los polifenoles inhiben potentemente la señalización de VEGFR-2 inducida por VEGF. La disminución de la densidad microvascular tras el efecto de los polifenoles, responde a que estos podrían actuar modulando las vías de señalización mediadas por los de VEGFR o modulando la disponibilidad de VEGF en angiogénesis de los fetos de pollo. Orsolic et al. (2016), demostraron que el AC es capaz de modular los niveles de VEGF disminuyéndolos en líquido ascítico, células tumorales y macrófagos dependiente de la dosis. En consecuencia, al bajar los niveles de VEGF disminuye la densidad microvascular, en una investigación realizada en animales.

Estudios anteriores se han centrado en los efectos de algunos polifenoles, Ahn et al. estudiaron la actividad antiangiogénica de AC y PC en conjunto con otros derivados del propóleo, demostrando que en ensayos con HUVEC in vitro e in vivo pueden suprimir el crecimiento tumoral. Además, Cuevas et al. (2015), informaron que los extractos etanólicos de propóleo chileno y Pinocembrina, uno de sus constituyentes principales, fueron capaces de modular la angiogénesis in vitro. Los estudios antes mencionados en conjunto con experimentación de material histológico se hacen necesario para corroborar, a través de microfotografías de las estructuras tisulares, lo ya visto por otros métodos de estudio de AC y PC en relación a la angiogénesis, a su vez, Wang et al. demostraron que el Ácido Caféico tiene el potencial de ser usado para minimizar el estrés oxidativo y las respuestas inflamatorias en enfermedades cardiovasculares y daño cerebral. En general, estudian su potencial efecto antioxidante, capacidad antiinflamatoria y otras actividades biológicos, pero en la actualidad hay pocas investigaciones que evalúen la capacidad antiangiogénica de estos polifenoles.
En conclusión, los polifenoles AC y PC demostraron tener capacidad antiangiogénica a nivel de angiogénesis fisiológica ya que disminuyen los niveles de expresión de VEGF y la densidad microvascular en corazón de pollo. Dicha capacidad no depende de la concentración de polifenoles utilizada, por lo tanto, se hace necesario seguir investigando el uso de estos polifenoles en más estudios asociados con angiogénesis fisiológica como patológica, para el beneficio de personas con patologías relacionadas con una formación excesiva de vasos sanguíneos, inhibición en la formación de los mismos o procesos cancerígenos en crecimiento tumoral.

OLIVARES, O. P.; RIQUELME, M. P.; LEIVA, A. P.; SALAZAR, L. A.; GUZMÁN-OYARZO, D. \& ROSAS, C. C. Anti-angiogenic effect of caffeic acid and pinocembrin in the process of physiological angiogenesis of chicken fetuses. Int. J. Morphol., 38(1):135-139, 2020.

SUMMARY: Angiogenesis is the process by which new blood vessels are formed from other existing ones. A balance between proangiogenic factors and anti-angiogenic factors within the microenvironment must exist for the process to be carried out correctly. Similarly, the existence of natural chemicals such as polyphenols, which are capable of being acquired in the diet, induce these factors in the angiogenic process. Polyphenols were administered in the methylcellulose filters on the of chorioallantoic membrane of White Leghorn eggs, maintaining the normal posterior development of the fetus. 15 chicken fetuses were fixed in buffered formalin, obtaining the hearts to histological processing, performing histological, histochemical and immunohistochemical techniques. VEGF levels and the ability of the blood vessels growing under the stimulation of the polyphenols were evaluated. Immunoreactivity was quantified by Image J. The results indicate that caffeic acid and pinocembrin decreased microvascular density and VEGF expression in hearts stimulated with these polyphenols. Both the caffeic and pinocembrin acids play an inhibitory role in the physiological angiogenesis process in the chicken heart, which decrease the microvascular density and could act by modulating the signaling pathways mediated by the VEGFR or by modulating the availability of VEGF. The use of these polyphenols could be useful in studies of other tissues associated with pathological angiogenesis.

KEY WORDS: Angiogenesis; Poliphenols; Caffeic acid; Pinocembrin.

\section{REFERENCIAS BIBLIOGRÁFICAS}

Ahn, M. R.; Kunimasa, K.; Kumazawa, S.; Nakayama, T.; Kaji, K.; Uto, Y.; Hori, H.: Nagasawa, H. \& Ohta, T. Correlation between antiangiogenic activity and antioxidant activity of various components from propolis. Mol. Nutr. Food Res., 53(5):643-51, 2009.

Birt, D. F.; Hendrich, S. \& Wang, W. Dietary agents in cancer prevention: flavonoids and isoflavonoids. Pharmacol. Ther, 90(2-3):157-77, 2001. 
Chung, A. S. \& Ferrara, N. Developmental and pathological angiogenesis. Annu. Rev. Cell Dev. Biol., 27:563-84, 2011.

Chung, A. S.; Lee, J. \& Ferrara, N. Targeting the tumour vasculature: insights from physiological angiogenesis. Nat. Rev. Cancer, 10(7):505-14, 2010.

Cuevas, A.; Saavedra, N.; Rudnicki, M.; Abdalla, D. S. \& Salazar, L. A. ERK1/2 and HIF1a are involved in antiangiogenic effect of polyphenolsenriched fraction from Chilean propolis. Evid. Based Complement. Alternat. Med., 2015:187575, 2015.

Diniz, C.; Suliburska, J. \& Ferreira, I. M. P. L. V. O. New insights into the antiangiogenic and proangiogenic properties of dietary polyphenols. Mol. Nutr. Food Res., 61(6), 2017. doi: 10.1002/mnfr.201600912

Fakhrejahani, E. \& Toi, M. Tumor angiogenesis: pericytes and maturation are not to be ignored. J. Oncol., 2012:261750, 2012.

Gerhardt, H.; Golding, M.; Fruttiger, M.; Ruhrberg, C.; Lundkvist, A.; Abramsson, A.; Jeltsch, M.; Mitchell, C.; Alitalo, K.; Shima, D.; et al. VEGF guides angiogenic sprouting utilizing endothelial tip cell filopodia. J. Cell Biol., 161(6):1163-77, 2003.

Li, J.; Zhang, Y. P. \& Kirsner, R. S. Angiogenesis in wound repair: angiogenic growth factors and the extracellular matrix. Microsc. Res. Tech., 60(1):107-14, 2003.

Manach, C.; Scalbert, A.; Morand, C.; Rémésy, C. \& Jiménez, L. Polyphenols: food sources and bioavailability. Am. J. Clin. Nutr. 79(5):727-47, 2004.

Mitjavila, M. T. \& Moreno, J. J. The effects of polyphenols on oxidative stress and the arachidonic acid cascade. Implications for the prevention/ treatment of high prevalence diseases. Biochem. Pharmacol., 84(9):1113-22, 2012.

Moyle, C. W.; Cerezo, A. B.; Winterbone, M. S.; Hollands, W. J.; Alexeev, Y.; Needs, P. W. \& Kroon, P. A. Potent inhibition of VEGFR-2 activation by tight binding of green tea epigallocatechin gallate and apple procyanidins to VEGF: relevance to angiogenesis. Mol. Nutr. Food Res., 59(3):401-12, 2015.

Orsolic, N.; Kunstic, M.; Kukolj, M.; Gracan, R. \& Nemrava, J. Oxidative stress, polarization of macrophages and tumour angiogenesis: Efficacy of caffeic acid. Chem. Biol. Interact., 256:111-24, 2016.

Rasul, A.; Millimouno, F. M.; Ali Eltayb, W.; Ali, M.: Li, J. \& Li, X. Pinocembrin: a novel natural compound with versatile pharmacological and biological activities. Biomed Res. Int., 2013:379850, 2013.

Rosas, C. C.; Roa, I.; Sinning, O. M.; Fuenzalida, B. M. \& Lemus, A. D. Celecoxib effect in a multiresistant variant of the TA3 tumor. A histological description. Int. J. Morphol., 31(2):392-398, 2013.

Rosas, C.; Sinning, M.; Ferreira, A.; Fuenzalida, M. \& Lemus, D. Celecoxib decreases growth and angiogenesis and promotes apoptosis in a tumor cell line resistant to chemotherapy. Biol. Res., 47(1):27, 2014.

Saad, M. A.; Abdel Salam, R. M.; Kenawy, S. A. \& Attia, A. S. Pinocembrin attenuates hippocampal inflammation, oxidative perturbations and apoptosis in a rat model of global cerebral ischemia reperfusion. Pharmacol. Rep., 67(1):115-22, 2015.

Ververidis, F.; Trantras, E.; Douglas, C.; Vollmer, G.; Kretzschmar, G. \& Paunopoulos, N. Biotechnology of flavonoids and other phenylpropanoid-derived natural products. Part I: Chemical diversity, impacts on plant biology and human health. Biotechnol. J., 2(10):121434, 2007.

Wang, Y.; Wang, Y.; Li, J.; Hua, L.; Han, B.; Zhang, Y.; Yang, X.; Zeng, Z.; Bai, H.; Yin, H.; et al. Effects of caffeic acid on learning deficits in a model of Alzheimer's disease. Int. J. Mol. Med., 38(3):869-75, 2016.

\author{
Dirección para correspondencia: \\ Dr. Carlos Rosas \\ Departamento de Ciencias Morfológicas \\ Facultad de Medicina y Ciencia \\ Universidad San Sebastián \\ Santiago \\ CHILE
}

Email: carlos.rosas@uss.cl

Recibido : 05-07-2019

Aceptado: 13-08-2019 